\title{
Multifractal dimension spectra in polymer physics
}

\author{
Ch. von Ferber ${ }^{1,2}$, Yu. Holovatch ${ }^{3}$ \\ 1 Institut für Theoretische Physik, Heinrich Heine Universität Düsseldorf, \\ D-40225 Düsseldorf, Germany \\ 2 School of Physics and Astronomy, Tel Aviv University, IL-69978 Tel-Aviv, \\ Israel \\ 3 Institute for Condensed Matter Physics \\ of the National Academy of Sciences of Ukraine, \\ 1 Svientsitskii Str., 290011 Lviv, Ukraine
}

Received September 4, 1998

We study the multifractal properties of diffusion in the presence of an absorbing polymer and report the numerical values of the multifractal dimension spectra for the case of an absorbing self avoiding walk or random walk.

Key words: multifractals, spectral function, Hölder exponent

PACS: $64.60 . A k, 61.41 .+e, 64.60 . F r, 11.10 . G h$

\section{Introduction}

The notion of non-integer dimension although developed by mathematicians in late 19 th - early 20th centuries has found its wide application in physics only in the last few decades. This application was mainly initiated by the pioneering work of B. Mandelbrot who attracted attention to the fact that "fractal" geometry provides an appropriate framework for the description of a whole range of complex structures that are formed from smaller subunits [1]. Whereas such structures are characterized by appropriate fractal dimensions, their growth and spatial correlations [2] are described by a (non-trivial) spectrum of multifractal (MF) dimensions [3,4].

In this paper, we study the properties of diffusion phenomena in the presence of an absorbing polymer. This provides another example of a multifractal phenomenon in condensed matter physics. To this end we use the model proposed by Cates and Witten [5] and derive the MF spectrum in the frames of a field theoretical formalism using the renormalization group [6,7] method. The MF spectrum is related to the spectrum of exponents governing scaling properties of copolymer stars [8]. We calculate this spectrum to the third order of perturbation 
theory and report the numerical values of the quantities that characterize the MF behaviour.

\section{The model and the multifractal measure}

Long flexible polymer chains [9] provide a classical example of fractal objects (see fig. 1a). Of particular interest is the manner in which such a chain absorbs diffusing

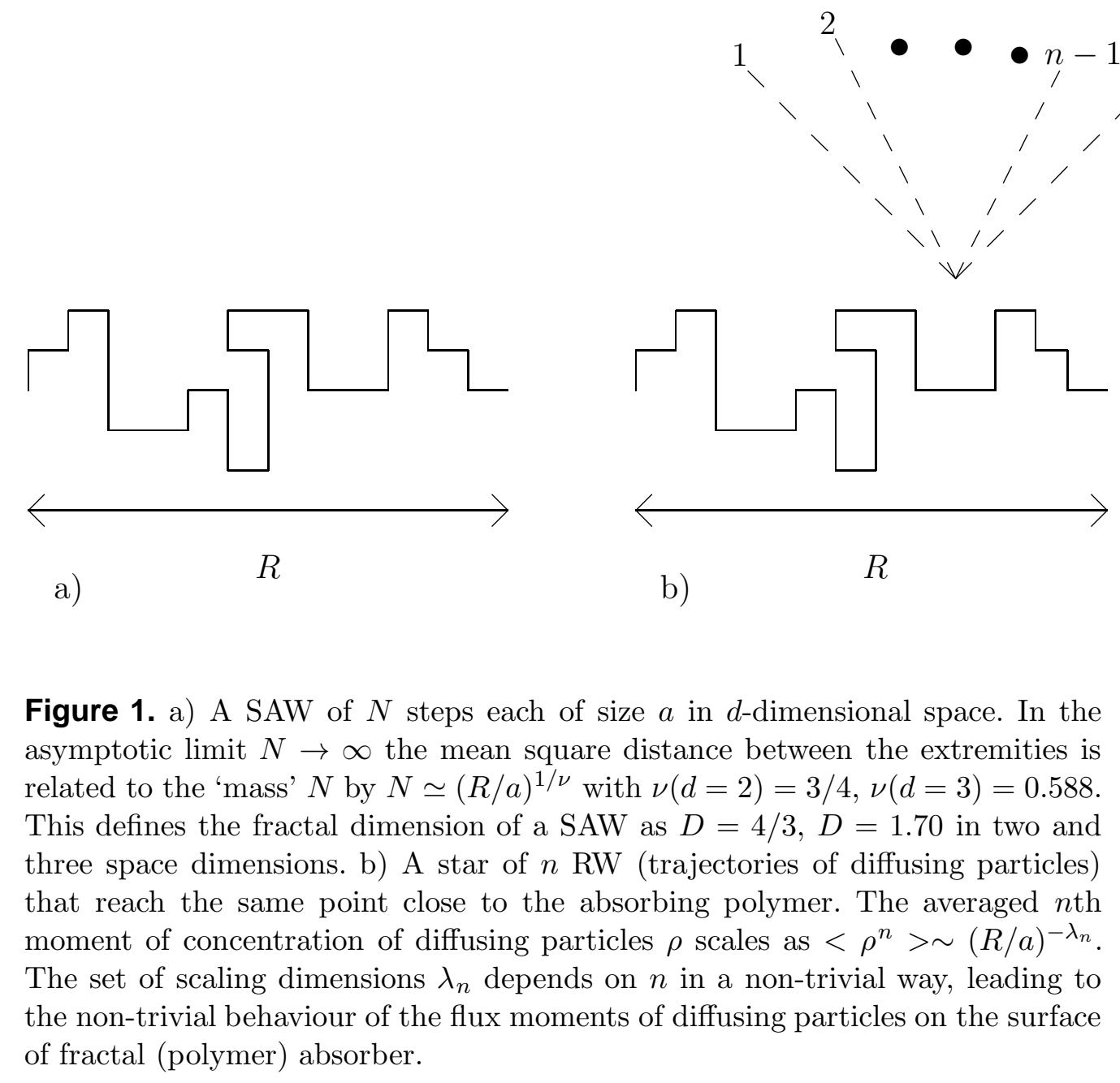

particles. The flux of particles on the surface of the absorbing chain is defined by the density of incoming particles close to the absorber (see fig. 1b). Taken $\rho(r)$ to be the probability density of incoming particles the problem is to solve the steady-state diffusion equation (Laplace equation)

$$
\nabla^{2} \rho(r)=0
$$

with boundary conditions for a given absorbing polymer

$$
\begin{aligned}
& \rho(r)=0 \quad \text { on the surface of the absorber, } \\
& \rho(r)=\rho_{\infty}=\text { const } \quad \text { for } \quad|r| \rightarrow \infty \text {. }
\end{aligned}
$$


One motivation for the study of this problem is to gain insight to the description of diffusion limited aggregation (DLA) [10]. The last phenomenon is much more complicated because of the fact that for DLA the boundary conditions are given on the surface of growing aggregate itself. The process described by equations (1), (2) may be rather considered as diffusion limited catalysis, when particles of one type interact with a prescribed fractal (a catalysing polymer) and transform into the other type [12].

Let the absorbing polymer of size $R$ be chosen from the well defined ensemble of SAW. Then we are interested in the moments of the field $\rho(r)$ close to the surface of the absorber (see fig. 1b). For distances $R \gg r>a$ ( $a$ being a cut off) the averaged moments $<\rho(r)^{n}>$ are expected to scale as [5]

$$
<\rho(r)^{n}>\sim(R / r)^{-\lambda(n)}
$$

where $\langle\ldots\rangle$ denotes the average over the ensemble of polymers. Let us take that the flux $\phi(x)$ onto any randomly chosen point $x$ of the absorber is proportional to the field $\rho(a)$ at a point that is as close as a cut off length $a$ from the absorber. Then, for the averaged moments of the flux one finds:

$$
<\phi^{n}>\sim(R / a)^{-\lambda(n)} .
$$

Following [5] we associate with the flux of the Laplacian field a "harmonic measure" $\mu(x)$ defined on the set of absorbing sites $x$ :

$$
\mu(x)=\phi(x) / \sum \phi(x),
$$

where the sum in (5) spans all sites $x$ of the absorber. In particular, the mass $M$ of the absorber is given by $\sum_{x} 1=M$ and (5) may be rewritten as

$$
\mu(x)=\phi(x) /(M<\phi>),
$$

where $\langle\ldots\rangle$ denotes the site average $\langle\ldots\rangle=\sum(\ldots) / M[11]$.

It is standard [3] to describe the properties of the harmonic measure by a set of exponents $D(n)$ given by:

$$
\sum \mu(x)^{n}=(R / a)^{(1-n) D(n)} .
$$

Note, that for $n=0 D(0)$ is the fractal dimension of the absorber. Multifractal scaling is found when the spectrum $D(n)$ is non-trivial: $D(n) \neq D(0)$, or correspondingly (c.f. equation (4)) $\lambda(n) \neq n \lambda(1)$. Note as well that from (4), (7) the exponents $\lambda(n)$ and $D(n)$ are related:

$$
n \lambda(1)-\lambda(n)=(1-n)[D(n)-D(0)] .
$$

In many cases the description of a multifractal measure is performed in terms of the Hölder exponent $\alpha$ and the spectral function $f(\alpha)$ that are defined by a Legendre transform of $D(n)[4]$ :

$$
\begin{aligned}
\alpha(n) & =\frac{\mathrm{d}}{\mathrm{d} n}[(n-1) D(n)], \\
f(\alpha) & =n \alpha(n)+(1-n) D(n) .
\end{aligned}
$$


The function $f(\alpha)$ differs for different fractal measures but it possesses certain 'universal' properties. In particular, its maximum $\max _{\alpha} f(\alpha)$ gives the fractal dimension of the absorber and $f$ is convex $f^{\prime \prime}(\alpha)<0$.

\section{The multifractal spectrum exponents and the spectral function}

The central idea that allows us to calculate the properties of the solutions of equation (1) with the boundary conditions (2) is that a path integral representation both for the field $\rho(r)$ and for the absorber (being a RW or a SAW) is possible $[5,12]$. In terms of the path integral solution of the Laplace equation one finds that $\rho(r)$ at point $r$ near absorber is proportional to the number of RW that end at point $r$ and avoid the absorber. The $n$th power of this field is proportional to the $n$th power of the above mentioned number, i.e. it is defined by a partition function of a star polymer with $n$ arms [13] (the latter is shown by dashed lines in fig. 1b). Furthermore, introducing the mutual avoidance conditions between the " $n$-arm star" and the 2-arm polymer (representing the absorber) one has to calculate the partition function of a co-polymer star consisting of chains of two different species that avoid each other. These correspond to the trajectories of diffusing particles (being RW) and the absorbing polymer (which for the purpose of present study is chosen as RW or SAW). Making use of the previously developed [8] theory of copolymer stars and networks and mapping the model of co-polymer stars to the appropriate field theory $[14,15]$ one may relate [17] the MF spectrum exponents (4) to the exponents that define the scaling properties of co-polymer stars. In particular, for a co-polymer star consisting of $n_{1}$ chains of species 1 and $n_{2}$ chains of species 2 the number of configurations $Z_{*}$ scales like [8]

$$
Z_{*} \sim(R / a)^{\eta_{n_{1} n_{2}}-n_{1} \eta_{20}-n_{2} \eta_{02}} .
$$

Here, the $\eta_{n_{1} n_{2}}$ represent the co-polymer star exponents. The latter have been calculated using a field theoretical renormalization group approach [6,7] and are known in the third order of perturbation theory in $\varepsilon=4-d$ and pseudo- $\varepsilon$ [18] expansions $[8,16]$. By means of a short-chain expansion [19] the set of exponents $\eta_{n_{1} n_{2}}$ can be related to the exponents $\lambda(n)(4)$, that govern the scaling of the $n$th moment of the flux onto an absorbing linear chain. Considering the absorber to be either a RW or a SAW we define the exponents

$$
\begin{aligned}
\lambda_{\mathrm{RW}}(n)^{G} & =-\eta_{2 n}^{G}, \\
\lambda_{\mathrm{SAW}}(n)^{U} & =-\eta_{2 n}^{U}+\eta_{20},
\end{aligned}
$$

here, we keep notations of $[8,16]$ for the expressions for exponents $\eta$ defined in different fixed points of the renormalization group transformation. Based on these expressions we get:

$$
\lambda_{\mathrm{RW}}(\varepsilon)=\varepsilon n-\frac{n(n-1) \varepsilon^{2}}{4}+\frac{n(n-1)(-1+n+3 \zeta(3)) \varepsilon^{3}}{8},
$$


Table 1. Exponents $\lambda_{\mathrm{RW}}(n)$ and $\lambda_{\mathrm{SAW}}(n)$ obtained in $\varepsilon$ and in pseudo- $\varepsilon$ expansion $(\lambda(\tau))$ techniques.

$$
\begin{array}{rlllll}
n & \lambda_{\mathrm{RW}}(\varepsilon) & \lambda_{\mathrm{RW}}(\tau) & \lambda_{\mathrm{SAW}}(\varepsilon) & \lambda_{\mathrm{SAW}}(\tau) \\
1 & 0.99 & 0.99 & 0.71 & 0.71 \\
2 & 1.77 & 1.81 & 1.31 & 1.33 \\
3 & 2.45 & 2.53 & 1.86 & 1.92 \\
& 4 & 3.01 & 3.17 & 2.34 & 2.44 \\
& 5 & 3.51 & 3.75 & 2.78 & 2.94 \\
& 6 & 3.95 & 4.28 & 3.19 & 3.41 \\
& \\
\lambda_{\mathrm{SAW}}(\varepsilon)= & \frac{3 \varepsilon n}{4}+\frac{n(7 n-18) \varepsilon^{2}}{128} \\
& +\frac{n\left(-149-42 n+108 n^{2}-276 \zeta(3)+540 \zeta(3) n\right) \varepsilon^{3}}{2048} .
\end{array}
$$

Here, $\varepsilon=4-d, d$ is the space dimension, and $\zeta(3) \simeq 1.202$ is the Riemann zeta function.

Using the perturbation expansions for the $\lambda$ exponents and the relations for $\lambda(n)$ and the spectral function some algebra leads to the corresponding expansions for $\alpha_{n}$ and $f\left(\alpha_{n}\right)$ :

$$
\begin{aligned}
\alpha_{\mathrm{RW}}(\varepsilon)= & 2+\frac{(-2 n+1) \varepsilon^{2}}{4}+\frac{\left(-4 n-3 \zeta(3)+3 n^{2}+6 \zeta(3) n+1\right) \varepsilon^{3}}{8}, \\
f_{\mathrm{RW}}(\varepsilon)= & 2-\frac{\varepsilon^{2} n^{2}}{4}+\frac{\left(2 n^{3}+3 \zeta(3) n^{2}-2 n^{2}\right) \varepsilon^{3}}{8} \\
\alpha_{\mathrm{SAW}}(\varepsilon)= & 2-\frac{\varepsilon}{4}+\frac{(7-36 n) \varepsilon^{2}}{128} \\
& +\frac{\left(-149-276 \zeta(3)-84 n+324 n^{2}+1080 \zeta(3) n\right) \varepsilon^{3}}{2048} \\
f_{\mathrm{SAW}}(\varepsilon)= & 2-\frac{\varepsilon}{4}-\frac{\left(18 n^{2}+11\right) \varepsilon^{2}}{128} \\
& +\frac{\left(216 n^{3}+540 \zeta(3) n^{2}-42 n^{2}-83+264 \zeta(3)\right) \varepsilon^{3}}{2048} .
\end{aligned}
$$

The exponents $\lambda(n)$ as well as the Hölder exponents $\alpha$ and the spectral functions $f(\alpha)$ in terms of the pseudo- $\varepsilon$ expansion [18] are given elsewhere $[17,20]$.

As is well known, the series of type (14)-(19), as they occur in field theory appear to be of asymptotic nature with zero radius of convergence. However, knowledge of the asymptotic behaviour of the series as derived from the renormalization group theory allows us to evaluate these asymptotic series (see e.g.[7]). To this end several procedures are available depending on the additional information known for the 
series to be resummed. We use the Borel summation techniques improved by a conformal mapping procedure that has served as a powerful tool in field theoretic calculations (see [21] for example).

Table 1 contains the results for the exponents $\lambda_{\mathrm{RW}}(n)$ and $\lambda_{\mathrm{SAW}}(n)$ obtained in $\varepsilon$ and in pseudo- $\varepsilon$ expansion techniques at $d=3$ from the corresponding values of exponents $\eta_{f_{1}, f_{2}}$ [8] with the application of this resummation procedure.

\section{Conclusions}

We have calculated the spectral function that describes the scaling of the moments of measure defined by diffusion near an absorbing polymer. These moments were calculated as averages over all configurations of the absorber instead of performing a site average. Thus the interpretation of $f(\alpha)$ itself does not directly correspond to the standard picture, where $f(\alpha)$ is interpreted as the fractal dimension of a particular subset of the measure [4]. Nevertheless we derive $f(\alpha)$ in the same way from the spectrum of scaling exponents of the moments of a measure. As we will see below in the general, properties of $f(\alpha)$ also hold for the present definition.

Our numerical results for the spectral function for space dimension $d=3$ are presented in Figs. 2a,2b. They were obtained from the series for $\alpha_{n}$ and $f\left(\alpha_{n}\right)$ as functions of $n$. We show the results of the resummation procedure described above applied to the series in $\varepsilon$ - and pseudo- $\varepsilon$-expansion. For comparison we also show the curve for direct summation of the $\varepsilon$ and pseudo- $\varepsilon$ series to the 2nd order (here we recover the second-order results of [5]). In addition we have performed an analytical continuation of our series in the form of a [2/1] Padé approximant for the third order series. It is obvious that the direct summation of the last series fails to converge and gives reasonable values for $\alpha_{n}, f\left(\alpha_{n}\right)$ only for small values of $n$, i.e. near the maximum of $f(\alpha)$ at $n=0$. The symmetry of the Padé approximant holds only in the region shown and may be an artifact of the method. On the left wing, where it coincides with the resummed results the Padé approximant provides a continuation that is compatible with the estimation for the minimal $\alpha$ value $\alpha_{\min }=d-2$. The Padé result is $\alpha_{\min }(\varepsilon)=1.333, \alpha_{\min }(\tau)=1.017$ for the RW absorber and $\alpha_{\min }(\varepsilon)=1.250$, $\alpha_{\min }(\tau)=1.013$ for the SAW absorber, which is calculated here only from 3rd order perturbation theory.

As can be extrapolated from the Padé approximant and as was also shown on the basis approximations for higher moments $n[5], f(\alpha)$ as it is defined here, will become negative near $\alpha_{\min }$ and $\alpha_{\max }$. For this reason the identification of $f(\alpha)$ as the fractal dimension of some identifiable subset is not possible here. Also the extrapolation of the resummed data seems to indicate such a behaviour. Note, however, that the perturbative approach, even in combination with resummation and analytical continuation is still not capable to give reliable results for high values of the expansion parameters. In particular this method is only good near the maximum of $f(\alpha)[22]$.

Though the results obtained for $\alpha_{n}$ and $f\left(\alpha_{n}\right)$ for a specific value of $n$ differ in both approaches, the same curve $f(\alpha)$ is described with better coincidence for the 
(Fig.2a)

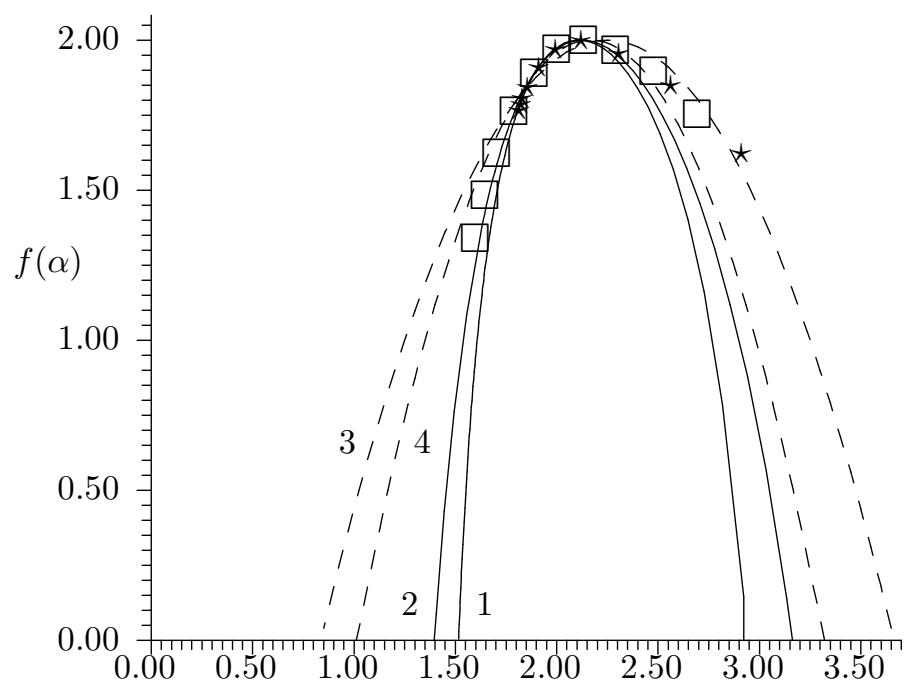

(Fig.2b)

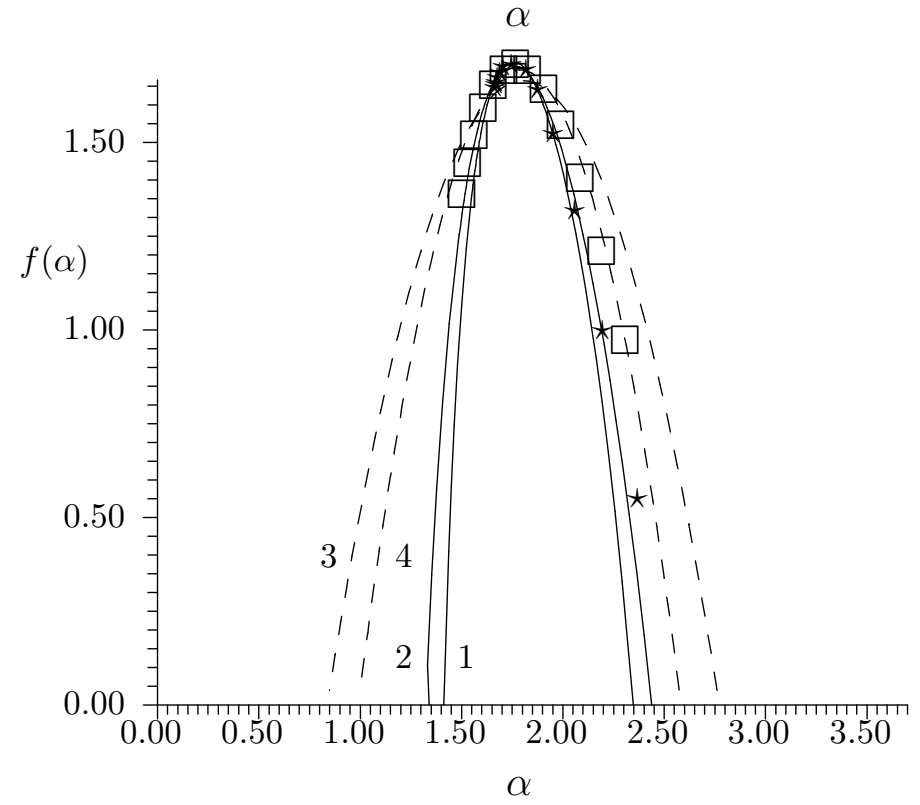

Figure 2. The spectral function $f(\alpha)$ for absorption on (a) RW and (b) SAW. Solid curves: 1 - [2/1] Padé approximant for $\varepsilon^{3}$ results, 2 - [2/1] Padé approximant for pseudo- $\varepsilon^{3}$ results; dashed curves: $3-\varepsilon^{2}$ results without resummation, $4-$ pseudo- $\varepsilon^{2}$ results without resummation; stars - resummed $\varepsilon^{3}$ results; boxes resummed pseudo- $\varepsilon^{3}$ results. 
left wing of the curves, corresponding to positive $n$. The resummation techniques we apply have proven to be a powerful tool already in the field theoretical approach to critical phenomena and have lead to high precision values for critical exponents. We believe that the application of these methods to calculations of MF phenomena also allows to improve the reliability and comparability of these results.

A generalization of our present approach to the study of spectral functions that describe the absorption at the core of an absorbing polymer star with a given number of arms will be published elsewhere.

It is our pleasure to acknowledge discussions with Lothar Schäfer, Bertrand Duplantier, Alexander Olemskoi. We are indebted to Gleb Oshanin for attracting our attention to the references [12]. Part of this work was supported by DFG (SFB 237) and the Minerva foundation.

\section{References}

1. Mandelbrot B.B. The fractal geometry of nature. New York, W.H.Freeman and Co, 1983.

2. Meakin P. The growth of fractal aggregates and their fractal measures. In: Phase transitions and critical phenomena. Ed. by C. Domb and J. L. Lebowitz. Vol. 12, New York, Academic Press, 1988. p. 336-442; Meakin P. Simulation of non-equilibrium growth and agregation processes. In: Springer Proceedings in Physics. Computer Studies in Condensed Matter Physics. Ed. by D. P. Landau, K. K. Moon, H.-B. Schüttler. Vol. 33, Berlin, Springer-Verlag, 1988. p. 55-64; Family F. Growth by gradients: fractal grows and pattern formation in a Laplacian field. ibid., p. 65-75.

3. Hentschel H.G.E., Procaccia I. The infinite number of generalized dimensions of fractals and strange attractors. // Physica D, 1983, vol. 8, p. 435-444.

4. Halsey T.C., Jensen M.H., Kadanoff L.P., Procaccia I., Shraiman B.I. Fractal measures and their singularities: The characterization of strange sets. // Phys. Rev. A, 1986, vol. 33 , No. 2, p. 1141-1151.

5. Cates M.E., Witten T.A. Diffusion near absorbing fractals: Harmonic measure exponents for polymers. // Phys. Rev. A, 1987, vol. 35, No. 4, p. 1809-1824.

6. Bogoliubov N.N., Shirkov D.V. Introduction to the Theory of Quantized Fields. New York, Wiley \& Sons, 1959.

7. Zinn-Justin J. Euclidean Field Theory and Critical Phenomena. New York, Oxford University Press, 1989.

8. von Ferber C., Holovatch Yu. Copolymer Networks: Multifractal dimension spectra in polymer field theory. // Europhys. Lett., 1997, vol. 39, No. 1, p. 31-36 (preprint cond-mat/9705273); von Ferber C., Holovatch Yu. Copolymer Networks and Stars: Scaling Exponents. // Phys. Rev. E, 1997, vol. 56, No. 6, p. 6370-6386 (preprint cond-mat/9705278v2).

9. For the purpose of present study polymer chains are considered as random walks (RW) or self-avoiding walks (SAW).

10. Witten Jr., T.A., Sander L.M. Diffusion-limited aggregation, a kinetic critical phenomenon. // Phys. Rev. Lett., 1981, vol. 47, No. 19, p. 1400-1403; Witten Jr., T.A., Sander L.M. Diffusion-limited aggregation. // Phys. Rev. A, 1983, vol. 27, No. 9, p. 5686-5697. 
11. In this study, the site average (6) is substituted by an ensemble average described by (3). Possible consequences of such substitution are discussed below.

12. Burlatsky S.F., Oshanin G.S., Likhachev V.N. Diffusion-controlled reactions involving polymer chains. // Sov. J. Chem. Phys., 1991, vol. 7(7), p. 1680-1698; Burlatsky S.F., Oshanin G.S. Diffusion-controlled reactions with polymers. // Phys. Lett. A, 1990, vol. 145, No. 1, p. 61-65; Oshanin G., Moreau M., Burlatsky S. Models of chemical reactions with participation of polymers. // Anv. Coll. Int. Sci., 1994, vol. 49, p. 1-46.

13. Duplantier B. Polymer network of fixed topology: renormalization, exact critical exponent $\psi$ in two dimensions, and $d=4-\epsilon$.// Phys. Rev. Lett., 1986, vol. 57, No. 8, p. 941-945; Duplantier B. Statistical mechanics of polymer networks of any topology. // J. Stat. Phys., 1989, vol. 54, No. 3/4, p. 581-680; Schäfer L., von Ferber C., Lehr U., Duplantier B. Renormalization of polymer networks and stars. // Nucl. Phys. B, 1992, vol. 374, No. 3, p. 473-495.

14. Schäfer L., Lehr U., Kappeler C. Higher order calculations of the renormalization group flow for multicomponent polymer solutions. // J. Phys. (Paris) I, 1991, vol. 1, p. 211-233.

15. von Ferber C., Holovatch Y., Schäfer L. Diffusion near an absorbing polymer. // Cond. Matt. Phys., 1996, iss. 7, p. 15-25.

16. von Ferber C., Holovatch Yu. Copolymer Networks: The spectrum of scaling dimensions. // Physica A, 1998, vol. 249, p. 327-331.

17. von Ferber C., Holovatch Yu., Field-theoretic operators for multifractal moments. In: Proc. of the 3rd Int. Conf. Renormalization Group'96, Dubna, 1997, p. 123-137 (preprint cond-mat/9705274).

18. Whereas the $\varepsilon$-expansion corresponds to collecting perturbation theory terms of the same powers of $\varepsilon=4-d$, in the pseudo- $\varepsilon$ expansion series at each power of the pseudo$\varepsilon$ parameter one collects contributions from the dimension-dependent loop integrals of the same order.

19. von Ferber C. Operator product expansion on a fractal: The short chain expansion for polymer networks. // Nucl. Phys. B, 1997, vol. 490, p. 511-542.

20. von Ferber C., Holovatch Y., preprint, 1998.

21. Le Guillou J. C., Zinn-Justin J. Critical exponents from field theory. // Phys. Rev. B, 1980, vol. 21, No. 9, p. 3976-3998.

22. The possible negative values of the spectral function were discussed already in [5] and a physical interpretation of $f(\alpha)$ was given as a histogram of the measure $\mu$ plotted in logarithmic variables. In this interpretation negative $f(\alpha)$ indicate that the number of sites with a certain logarithmic measure $\alpha \sim \ln \mu$ decreases as the size of the absorber $R$ increases. Thus for large $R$ this number can only be defined by an ensemble average, as performed here. 


\title{
Спектр мультифрактальних вимірностей у фізиці полімерів
}

\author{
К. Фон Фербер ${ }^{1,2}$, Ю. Головач ${ }^{3}$
}

1 Інститут теоретичної фізики, Університет Гайнріха Гайне, D-40225 Дюссельдорф, Німеччина

2 Фізичний факультет, Університет Ессен, D-45117 Ессен, Німеччина

3 Інститут фізики конденсованих систем НАН України, 290011 Львів, вул. Свєнціцького, 1

Отримано 4 вересня 1998 р.

Досліджені мультифрактальні властивості дифузії поблизу фрактального (полімерного) абсорбера та приведені числові значення спектру мультифрактальних вимірностей для випадку абсорбера у вигляді блукань із самоуниканням та випадкових блукань.

Ключові слова: мультифрактали, спектральна функція, показник Гельдера

PACS: $6460 . A k, 61.41 .+e, 64.60 . F r, 11.10 . G h$ 